
allemande

51-1 | 2019

La République démocratique allemande dans l'espace public européen (1949-2018)

\title{
Die DDR retten? Historisierung der DDR-Diktatur in französischen Medien 1989-2009
}

Dominique Herbet

\author{
(2) OpenEdition \\ Journals \\ Édition électronique \\ URL : https://journals.openedition.org/allemagne/1565 \\ DOI : 10.4000/allemagne. 1565 \\ ISSN : 2605-7913 \\ Éditeur \\ Société d'études allemandes \\ Édition imprimée \\ Date de publication : 2 juillet 2019 \\ Pagination : 129-140 \\ ISSN : 0035-0974 \\ Référence électronique \\ Dominique Herbet, „Die DDR retten? Historisierung der DDR-Diktatur in französischen Medien \\ 1989-2009", Revue d'Allemagne et des pays de langue allemande [Online], 51-1 | 2019, Online erschienen \\ am: 02 Juli 2020, abgerufen am 03 Juni 2022. URL: http://journals.openedition.org/allemagne/1565; \\ DOI: https://doi.org/10.4000/allemagne.1565
}




\section{Die DDR retten? Historisierung der DDR-Diktatur in französischen Medien 1989-2009}

\section{- Dominique Herbet*}

Die im Titel vorliegender Studie formulierte Fragestellung soll keine Provokation an sich sein, hier wird aber absichtlich eine Losung aufgegriffen, die im November und Dezember 1989 Vorhaben und Haltung der Bürgerbewegung in der DDR und darunter vieler Intellektuellen bzw. Dissidenten in der DDR reflektierte: durchaus im Geiste des von Stefan Heym im Fernsehen vorgelesenen Aufrufs „Für unser Land“ vom 28. November und seiner Mahnung, die DDR werde ja schließlich zu einer „Fußnote der Weltgeschichte“.

Es soll nämlich um das Bild der DDR in Frankreich nach 1990 gehen, also auch im Vergleich mit einem eher westdeutsch geprägten DDR-Bild sowie in der Perspektive der Post Cold War Studies: hat sich zum Beispiel das Bild des SED-Staates im französischen öffentlichen Raum gewandelt? Dabei muss davon ausgegangen werden, dass es nicht nur ein Bild der DDR und der DDR-Bürger gab und gibt. In der Bundesrepublik wurde seit der Jahrhundertwende darüber heftig gestritten, ob das vorrangig und intensiv erforschte Bild des SED-Staates einem nuancierten Bild der Ostdeutschen in der Diktatur weichen sollte. Le Monde verwies in diesem Zusammenhang auf eine besondere Sichtweise der französischen Historiker und bezog sich dabei auf Sandrine Kott $^{(1)}$ : sie erwähnte nämlich „die Stellung des Außenseiters“, „die Wichtigkeit der kommunistischen Kultur im öffentlichen Raum in Frankreich“, „French Touch“ also, der „sozio-historische Perspektiven vorziehe“ und sich so einer „allzu politischen und monolithischen Auffassung der kommunistischen Regimes entzöge“.

Und es gibt auch keine einzige homogene Öffentlichkeit, wie Pierre Bourdieu es lapidar formuliert hat: „Die Öffentlichkeit existiert nicht.“ Der Wissenschaftler müsse dann „versuchen, sie in ihrer Vielfalt zu erfassen, der Diversität der Individuen und der gesellschaftlichen Gruppen entsprechend“, was Pierre Laborie auch bestätigte: „Die

* Professor der Germanistik an der Universität Lille, CECILLE EA 4074.

1 Nicolas Offenstadt, „Au pays du socialisme réel, les historiens français férus de RDA“, Le Monde des livres, 28. November 2014, S. 3. 
Öffentlichkeit ist vielfältig und wechselhaft“(2). Im deutsch-französischen öffentlichen Raum, der sich vor allem seit Anfang des 21. Jahrhunderts immer stärker strukturiert hat ${ }^{(3)}$, spielen Medien eine große Rolle, wobei „Funktionsmängel der Medienöffentlichkeit“ „im Anschluss an Habermas' kritischem Befund“ festgestellt wurden. "Alternativ- und Gegenöffentlichkeit“ - im Sinne von Teilöffentlichkeit - sind dann entstanden $^{(4)}$. Öffentlichkeit wird dann als „Netzwerk für die Kommunikation von Inhalten und Stellungnahmen“ beschrieben, wobei diese Netzwerke über eigene Foren in den Medien verfügen. Bei Medienöffentlichkeit ${ }^{(5)}$ darf nämlich die Frage des Feed back nicht außer Acht gelassen werden, da Zeitungen zum Beispiel - also Medien - „jeweils nur Teile einer Gesellschaft erreichen und jede noch so einseitige mediale Darstellung historischer Ereignisse verschiedene Interpretationen der Rezipienten zulasse“(6). Als Beispiel eines Wandels der öffentlichen Meinung bzw. einer Gegenöffentlichkeit in Frankreich ist die Biermann-Affäre tradiert, die ja durchaus von der Präsenz der DDR zeugte und die bis nach Frankreich Wellen geschlagen und die Kreise verstimmt hat, die bisher das sozialistische Deutschland immer verteidigt hatten. Die Vereine und Zeitschriften, die mit der DDR sympathisierten, nahmen kritisch Abstand zur Ausbürgerung des Liedermachers Wolf Biermann im November $1976^{(7)}$. In Le Monde erschien im Dezember 1976 ein Beitrag des Schriftstellers Raymond Jean, Mitglied der französischen Kommunistischen Partei (Parti communiste français - PCF) und des Freundschaftsvereins France-RDA. Er kritisierte die Ausbürgerung von Biermann und prangerte die stalinistische Praxis der DDR an ${ }^{(8)}$. Die Germanistin A.-M. Pailhès fuhr fort: „Im Jahre 1977 sorgte dann der Fall Bahro für Aufregung. L'Humanité veröffentlichte Artikel gegen die Inhaftierung des Philosophen und Autors der Alternative." Die brutale Unterdrückung passte tatsächlich nicht zum positiven Bild der DDR als Modell und als Alternative zum westdeutschen Staat oder zum kapitalistischen System in westlichen Demokratien überhaupt.

Vorliegende Studie artikuliert sich vorwiegend um drei Hauptmomente: 1989-1990 wird als Referenz in der Berichterstattung genommen; um die Jahrhundertwende 1999/2000 wurde zuerst Bilanz über die Verwirklichung der inneren Einheit gezogen, während 2009 dann die Gelegenheit zu doppelten Feierlichkeiten zum 60. Jahrestag der Gründung der Bundesrepublik und 20. Jahrestag des Mauerfalls bot. Daher wird vorwiegend chronologisch verfahren.

2 Pierre Bourdieu, Questions de sociologie, Paris, Éditions de Minuit, 1981, S.224; Pierre Laborie, L'opinion française sous Vichy, Paris, Le Seuil, 1990, S. 41.

3 Dominique Herbet, „Les médias français en campagne pour les élections au Bundestag de septembre 2017“, Allemagne d'aujourd'hui, 223 (2018), S. 95-107. Klaus BecK, Kommunikationswissenschaft, Konstanz, UVK, 2007, S. 108.

5 Ebd., S. 111-113.

6 S. Gerrit Dworoks, Rezension über Martin Stallmann, Die Erfindung von Mai 68, Göttingen, Wallstein, 2017, www.hsozkult.de/publicationreview/id/rezbuecher-28186.

7 Anne-Marie PAIlhès, „Die Beziehungen zwischen der DDR und Frankreich in den 70er und 80er Jahren: zwischen Herabsetzung und Idealisierung“, La Clé des Langues (en ligne), Lyon, ENS de LYON/ DGESCO (ISSN 2107-7029), Juni 2014, http://cle.ens-lyon.fr/allemand/civilisation/die-beziehungenzwischen-der-ddr-und-frankreich-in-den-70er-und-80er-jahren-zwischen-herabsetzung-und-idealisierung (letzter Abruf: 04.08.2018).

8 Ebd.; Raymond JeAn, „Communiste critique“, Le Monde, 10. Dezember 1976, S. 3. 
Ab 1999 ging es in den deutschen und französischen Medien vorrangig um Geschichtspolitik im wiedervereinigten Deutschland. „Die DDR retten“ bedeutete dann eher, für eine Geschichtspolitik zu kämpfen, die nicht nur die Erinnerung an die Diktatur und die Delegitimierung des SED-Systems zum Ziel hatte. Drei Haupttendenzen lassen sich bei dem umfangreichen Korpus definieren, vorwiegend Artikeln aus überregionalen Tageszeitungen, Referenzmedien wie Le Figaro, L'Humanité, Le Monde und Libération, die insgesamt die französische gebildete Öffentlichkeit widerspiegeln. Genauso wie in der Bundesrepublik wurde zuallererst regelmäßig an das Ende der Diktatur, den Fall der Berliner Mauer und des Eisernen Vorhangs erinnert. Zweitens zeigt der Korpus auch, dass die von S. Heym befürchtete Einstufung der DDR-Geschichte in die Regionalgeschichte im Falle der Kulturgeschichte in den Medien zwischen 1989 und 2009 - und sogar darüber hinaus - nicht eingetroffen ist, was 2009 angesichts der Kontroverse um die Ausstellung „60 Jahre 60 Werke“ in der Bundesrepublik nicht eindeutig schien ${ }^{(9)}$. Schließlich fällt auf, dass sich die französischen Medien intensiv mit der Ostalgie-Welle beschäftigten, vielleicht nicht nur um der Ostalgie willen, sondern um eine neue Sichtweise über den real existierenden Sozialismus zu fördern, was den vorausgehenden Ausführungen durchaus gerecht werden könnte. Dabei wird zwei Fragenkomplexen nachgegangen: inwiefern unterscheidet sich die Linie der Referenzzeitungen von einer mehrheitsfähigen Linie in den deutschen Medien? Lässt sich wegen des politischen Gewichts der PCF (bis 1963 und sogar bis 1983) eine Sehnsucht nach einem kommunistischen Modell feststellen?

\section{Ein historisches Weltereignis und drei Hauptthemen: Mauerfall und Ende der Teilung, Ende der Diktatur und absoluter Sieg des Kapitalismus}

Fast 30 Jahre nach dem Mauerfall muss daran erinnert werden, dass der Fall der Mauer 1989 ein hoffnungsträchtiges Weltereignis gewesen ist: die Bilder der jubelnden Menschen auf der Mauer haben sich in allen Ländern der westlichen Welt dem kollektiven Gedächtnis als symbolische Verkörperung einer neuen freien Welt eingeprägt, weil die Mauer der Schande für die „freie Welt“ Symbolkraft hatte. Das fasste die konservative Zeitung Le Figaro 1999 so zusammen ${ }^{(10)}$ :

„An dem Jahrestag des Mauerfalls in Berlin wäre es ungehörig, sich nicht der Freude hinzugeben. Man dürfe ja nicht vergessen, dass am 9. November 1989 Millionen von Menschen die Freiheit erkämpft haben und der Teilung Europas ein Ende gesetzt wurde. Man dürfe auch nicht vergessen, dass an jenem Tage Marxismus-Leninismus und Planwirtschaft definitiv in den Müll der Geschichte gelangten."

Das wollte L'Humanité (Organ der PCF - 49.500 Exemplare 2016) 2016 noch nicht einsehen und die Redaktion lieferte folgende Analyse in Bezug auf die Ereignisse der Herbstrevolution, die ja schon 1989 von der Zeitung vertreten worden war: die PCF habe damals Gorbatschows Politik unterstützt, obwohl oft das Gegenteil behauptet

9 Dominique Herbet, „Dunkeldeutschland, Nullhoffnungszone“: quelle image la presse allemande véhicule-t-elle à la veille des vingt ans de la chute du mur?“, Allemagne d'aujourd'hui, 189 (2009), S. 217-228.

10 Marie-France CAlle, Jean-Pierre Robin, „La réunification dix ans après...“, Le Figaro, 9. November 1999, S. 1-2. 
worden sei (so die Redaktion der Zeitung), und habe Honeckers Haltung als befremdend empfunden ${ }^{(11)}$. Dessen Starrsinn habe nämlich dafür gesorgt, dass

„die DDR, die eins der am weitesten entwickelten sozialistischen Länder war und wo der Kommunismus historisch überhaupt Sinn machte - der heutige Einfluss der Linkspartei zeugt ja davon - am Ende auch für das Scheitern Mitverantwortung trug. Der Kommunismus in der DDR war doch keine aufgezwungene Diktatur. Es existierten Kräfte, die für einen demokratischen Sozialismus eintraten“.

Und der Autor, ein Historiker, schlussfolgerte: so hätten „die mächtigen reaktionären Kreise in Deutschland und auf der Welt genug Handlungsraum gehabt, um einen grenzlosen Kapitalismus aufzuzwingen, in dem wir heute leben“. Er sei „noch davon überzeugt, dass eine andere Geschichte hätte geschrieben werden können und dass L'Humanité vom 11. November 1989 vom Machbaren gezeugt habe“. Diese Schlussfolgerung hätte ein ehemaliger nostalgischer SED-Kader ziehen können, nur hätte er Michael Gorbatschow und/oder Egon Krenz für den sogenannten Ausverkauf der DDR verantwortlich gemacht ${ }^{(12)}$. Die Gefahr solcher Thesen ist, dass sie dem Revisionismus nahe sind. Aber die Frage des wirtschaftlichen Modells war ja relevant für die französische Öffentlichkeit, wo der Einfluss der Kommunisten zwar seit 1983 stark zurückgegangen war, derjenige der Sozialisten bis 2017 relativ groß war. Im Organ der PCF war auch ein Beitrag über die Arbeit der Treuhandanstalt zu lesen ${ }^{(13)}$, die es für den „Ausverkauf“ der DDR-Betriebe/ Kombinate verantwortlich machte. Westdeutsche Investoren und „Kriminelle“ hätten ja Nutzen aus „undurchsichtigen“ Geschäften gezogen, wie es die Kohl- und Elfaffären bewiesen hätten. Der Diskurs um „Skandale“, Geldwäsche“, „Plünderung“ „Enteignung der Ostdeutschen“ orientierte sich an den Argumenten der deutschen Revisionisten in der Linkspartei ${ }^{(14)}$. Mit L'Humanité muss also von einer tradierten Gegenöffentlichkeit ausgegangen werden, die ja weder den Historikern noch den Referenzmedien zutraut, ein authentisches Bild der DDR zu vermitteln und so den Thesen von dem Linksflügel der PDS und ihrer Presse (Junge Welt zum Beispiel) nahe ist.

Die linksliberale Zeitung Le Monde hat schon 1994 andere Töne geschlagen; deren berühmter Korrespondent Henri de Bresson ${ }^{(15)}$ lobte vor der Bundestagswahl die fast „blühenden Landschaften“ in Cottbus in der Lausitz. Der Bürgermeister gehörte der CDU an und arbeitete dennoch mit der PDS (28\% der Stimmen) zusammen. Dank der Braunkohleförderung lag die Arbeitslosigkeit im Jahre 1994 bei etwa 12\% in der Gegend und sogar $8 \%$ in Cottbus gegen einen Durchschnitt von 15,5\% in den neuen Bundesländern. Und vor allem betonte Bresson, die Cottbusser hätten ja Gelegenheit gehabt, das Erreichte ganz genau zu ermessen und zu sehen, wie sehr das Land im Gegensatz zu

11 Frederick Genevée, „La chute du mur de Berlin“, L’Humanité, 30. August 2016. 2003 verfasste Genevée eine Doktorarbeit unter dem Titel: „Le PCF et la justice: des origines aux années cinquante, organisation, conceptions, militants et avocats communistes face aux normes juridiques" (Betreuer der Doktorarbeit: Serge Wolikow, Dijon). Er ist Mitglied der kommunistischen Partei.

12 Dominique Herbet, „DDR-Erinnerungsdiskurs in der Monatszeitschrift Rotfuchs“, in: Carola Ḧ̈HNEL, Elisa Goudin (Hg.), Narrative ostdeutscher Erinnerung, Berlin, Frank \& Timme, 2011, S. $127-144$. Bruno Odent, „La Treuhand, la source aux scandales“, L’Humanité, 25. Januar 2000. 
Polen von den Transferleistungen des Bundes sowie der Europäischen Union Nutzen gezogen hat. Les Échos sprach 1996 ebenfalls Klartext: in ihrer Beilage zog die Redaktion Bilanz und das Fazit fiel drastisch aus, da die DDR ,innerhalb eines Jahres von der Schwerindustrie des 19. Jahrhunderts zur globalisierten Wirtschaft vom Anfang des 21. Jahrhunderts“(16) übergegangen war. Die Lage im Industrie-Sektor vor der Wende war so desolat, dass das Land sowieso zusammengebrochen wäre: dem SED-Regime sei es gelungen, sogar die CIA reinzulegen, da das BIP völlig überschätzt worden sei (um 33\%). Die Redaktion leugnete die Folgen des Umtauschkurses nicht, hob aber die Sanierungen hervor und betonte schließlich das hohe Bedürfnis nach Subventionen, um neue Branchen entwickeln zu können. Le Figaro stimmte 1999 überein und bezog sich auf den ehemaligen amerikanischen Botschafter in Berlin, Vernon Walters, der die DDR 1989 für „eine lebendige Leiche“ hielt ${ }^{(17)}$. Zwar gestand die Redaktion Fehler des Christdemokraten Helmut Kohl ein, der den DDR-Bürgern mit dem Wechselkurs von 1:1 ein "giftiges Geschenk“ gemacht habe, aber dies sei eben notwendig gewesen, um dem „Massenexodus“ vorzubeugen ${ }^{(18)}$. Zwar sei trotz Transferleistungen in Milliardenhöhe die Arbeitslosigkeit doppelt so hoch geblieben wie in West-Deutschland, dennoch seien „sogar Arbeitslose besser dran als zu DDR-Zeiten“. 2009 veröffentlichte Le Figaro einen Beitrag über „Züge Richtung Freiheit“(19) und dokumentierte den „Exodus“ im Kontext der Perestroika in der UdSSR und der Weltentspannung überhaupt. Dabei folgte der Journalist dem Leitmotiv der Einheitsfeier in der Bundesrepublik „Freiheit“ sowie von Merkels Rede: „Das Jahr 1989 hat Geschichte geschrieben“ genauso wie „zigtausende Deutsche“, die der Einheit den Weg geebnet hatten.

Die Aufarbeitung der DDR-Diktatur wird aber - rein statistisch gesehen weniger ausführlich behandelt als in der deutschen Presse. Französische Medien interessieren sich dennoch manchmal für heikle Fragen und liefern nicht nur Mainstream-Berichte. Im Jahre 1999 warf Le Monde die Frage der Amnestie für die

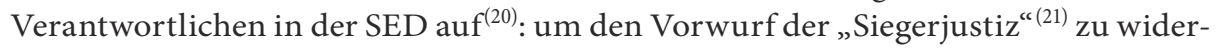
legen, der von Egon Krenz angeführt wurde, fasste die Zeitung die Stellungnahmen der deutschen Politiker und Referenzmedien zusammen. Der Artikel erwähnte die Empörung in Bonn (Angela Merkels z.B.) und in der Bevölkerung, wo vorwiegend der Opfer gedacht wurde, namentlich der 900 Deutschen, die auf der Flucht ermordet wurden. Er verwies auf Lothar de Maizières Stellungnahme (CDU), des letzten Ministerpräsidenten der DDR, der das Strafverfahren für gescheitert hielt, da viele Täter wie Markus Wolf zum Beispiel schweigen würden, solange sie straffällig waren. „98\% der 1990 eingeleiteten 22.500 Strafverfahren sind abgeschlossen“ und

16 Vincent de Feligonde, „Ex-RDA: le coup de blues“, Les Échos, 30. September 1996, S. 33.

17 Jean-Jacques Mével, „Dix ans après la chute du mur, les grands témoins racontent (2)“, Le Figaro, 4. November 1999, S. 4.

18 Calle/Robin, „La réunification dix ans après“ (Anm. 10).

19 Jean-Jacques Mével, „Les trains de Prague vers la liberté. Pendant tout l'été des brèches s'étaient ouvertes dans le bloc soviétique“, Le Figaro, 1. Oktober 2009, S. 2.

20 Arnaud Leparmentier, „Une amnistie pour réconcilier les Allemands?“, Le Monde, 26. Januar 1999, S. 18.

21 Das Wort „Siegerjustiz“ ist negativ konnotiert und weist auf die Verfolgung der NS-Verbrechen und die Nürnberger Prozesse in der unmittelbaren Nachkriegszeit hin. 
nur E. Krenz wurde zu sechseinhalb Jahren Haft verurteilt. Honecker und Mielke konnten aus gesundheitlichen Gründen und Altersgründen nicht zur Rechenschaft gezogen werden. Nur $1 \%$ der Verantwortlichen wurde schließlich verurteilt. Die Bilanz war dann eher ökonomischer Natur in Le Monde: Wolfgang Thierse (SPD ex-DDR-Bürger) hielt die Amnestie für sinnlos, „um die innere Einheit zu fördern“, die Politik solle eher für "Chancengleichheit“ sorgen, indem Stellen, Lehrplätze, Karrieremöglichkeiten in Verwaltung, Wirtschaft und Forschung" geschaffen werden. Die Medien riefen also eher ein nuanciertes Bild wach, was sich vielleicht dadurch erklären lässt, dass Deutschland bis 2005 der kranke Mann in der EU gewesen ist, da das ersehnte Wirtschaftswunder nach der Einheit nicht eingetreten ist. Infolgedessen und parallel dazu blieb das Interesse für ostdeutsche Kultur eher rege.

\section{DDR-Kultur und keine Fußnote der europäischen Kulturgeschichte}

1990 fokussierte zum Beispiel Le Monde auf die Zukunft der DEFA (Deutsche Film $A G)^{(22)}$ : sie hob eine gesamtdeutsche Perspektive hervor, da die Vorsitzenden des Filmvereins Elke Misselwitz und Rolf Richter die westdeutschen Kollegen um Hilfe gebeten hatten, um jedwede Demontage abzuwenden. Und sie wollten sogar in Brüssel und in Paris bei Jack Lang um Unterstützung bitten, was die besondere Stellung der DDR-Kultur im öffentlichen europäischen Raum belegen kann. Dabei unterstrich die Zeitung das Erbe der DEFA, der Journalist schwieg die Gleichschaltung des kulturellen Schaffens in der DDR tot.

Werke ostdeutscher Schriftsteller konnten auch zur Weltliteratur gerechnet werden und nicht nur in der Perspektive des Kalten Krieges. Am 1. Oktober 1990 veröffentlichte Le Monde Diplomatique ein Dossier über die ostdeutsche Kulturlandschaft ${ }^{(23)}$ : Christa Wolfs Werk wurde in einer langen Ausführung vorgestellt, die Polemik über ihre Haltung unter dem SED-Regime eingeschlossen, die sich im Westen nach der Wende entfacht hatte. Die Zeitschrift bezog sich auf den Kulturredakteur Jürgen Serke, der in ihrem Werk Was bleibt nur „Weinerlichkeiten einer Heuchlerin für andere HeuchlerInnen“ sah. Dennoch spreche Wolfs Werk im Allgemeinen auch für ihr Engagement gegen das Regime. Die Zeitschrift ging interessanterweise davon aus, dass ihre französische Leserschaft über genaue Kenntnisse im Literatur- und Kulturbereich verfügte. Denn dann wurde auch auf die Säuberung der Kulturszene in den fünfziger Jahren hingewiesen, mit den emblematischen Fällen von Walter Janka und Walter Harich, die durch das SED-Regime verfolgt und ausgeschlossen wurden. Aber Hauptthema waren die Zukunftsperspektiven einer stark subventionierten Filmbranche: die Gefahr einer „Vereinnahmung“ im Kontext einer konkurrenzvollen Landschaft wurde betont. Schließlich fragte der Journalist nach der Zukunft des Pariser Kulturinstituts, das „die Schwierigkeiten im eigenen Land nie verhehlte“.

22 Henri de Bresson, „Berlin ville ouverte. Espoirs et inquiétudes des artistes dans l’Allemagne réunifiée. Studios en péril“, Le Monde, 2. August 1990, S. 11.

23 Jean-Michel Palmier, „Surmonter le passé, abolir les préjugés“, Le Monde diplomatique, 1. Oktober 1990, S. 4-5. 
Le Monde interessierte sich dann im Oktober 1994 für das Programm der Berliner Theater und bezog sich auf Brechts Erbe ${ }^{(24)}$. In der Tat galt das Interesse oft fast ausschließlich Berlin, manchmal waren kritische Töne zu hören: so zum Beispiel in Libération $^{(25)}$, als Daniel Barenboim die magere Unterstützung des Bundes für Kultur und Musik bemängelte. Der Journalist erwähnte, dass der emblematische ostdeutsche Regisseur

„Heiner Müller sehr enttäuscht gewesen ist, dass Berlin die einmalige Chance nicht ergriffen hatte, ein neues System auf halbem Wege zwischen Kapitalismus und Kommunismus zu entwickeln und von der Euphorie zur Apathie übergegangen war. Es ist an der Zeit, nach vorne zu blicken und eine neue gemeinsame Kultur zu erfinden“.

Die Rezension in L'Humanité über Stefan Heyms Les Architectes (Die Architekten) ließ sich durchaus in diese Tendenz der Aufwertung der ostdeutschen Kultur und in die eben analysierte Linie dieser Zeitung einreihen ${ }^{(26)}$ : Die Handlung des Romans spielt sich im Kontext von Chruschtschows Bericht über Stalin ab. Die Hauptfigur, Arnold Sundstrom, Kommunist und berühmter Architekt, war mit der Gestaltung eines emblematischen sozialistischen Projektes beauftragt worden: die Straße des Weltfriedens. Es stellte sich aber heraus, dass seine Ästhetik an Speers Ästhetik erinnerte, und dazu wurde er des Stalinismus bezichtigt, sowie an den Säuberungen teilgenommen zu haben: anstatt den Fall zu bearbeiten, hatte sich die Führung für einen „schlechten“ Kompromiss entschieden. Der Leser der Architekten könne leicht einsehen, warum „die Substanz der Erneuerung unterwegs verlorenging“, was unter der Feder von S. Heym, einem berühmten Dissidenten, der für die PDS in den ersten gesamtdeutschen Bundestag gewählt wurde, glaubwürdig erschien. Wusste der französische Leser Bescheid? War da nicht eher der Trend zur Instrumentalisierung durch Vertreter des Eurokommunismus am Werk (Reformer innerhalb der westlichen kommunistischen Parteien vor Ende des Kalten Krieges)?

1998 analysierte die linke Tageszeitung Libération den sogenannten Erinnerungsboom in den neuen Bundesländern ${ }^{(27)}$. Sie begrüßte die Tatsache, dass „sich Ostdeutschland unter all den ehemaligen osteuropäischen Diktaturen so schnell und so intensiv für eine oft masochistische Aufarbeitung der Vergangenheit engagierte“, was sie auf die Konfrontation von zwei unterschiedlichen Regimes“ zurückführte. Sie listete die bekanntesten Erinnerungsorte auf, Hohenschönhausen, Marienborn, die Ostberliner Stasizentrale, so wie eine Sammlung aller in der DDR im Auftrag gegebenen Werke in der Burg von Beeskow. In Eisenhüttenstadt hatte sogar ein westdeutscher Historiker ein Alltagsmuseum geschaffen, in dem Gegenstände des alltäglichen Bedarfs - eigentlich durch DDR-Bürger ausgewählte Gegenstände, die sie als typisch für die DDR betrachten - zusammengetragen und ausgestellt wurden: „Geschichte ist nicht nur Historie, es sind auch die kleinen Geschichten, die man sich selbst erzählt.“ Die Redakteurin

24 Brigitte SALINo, „La rentrée théâtrale sur les scènes berlinoises. Edward Bond et Heiner Müller sous l'œil de Brecht“", Le Monde, 4. Oktober 1994, S. 19.

25 Éric DAHAN, „Berlin est passé de l'euphorie à l’apathie“, Libération, 3. Juni 1998, S. 39.

26 François Eychart, „1956 en Allemagne. Une mise à nu des réalités de la RDA“, L’Humanité, 2. Februar 2008; Stefan Heym, Les Architectes (Vorwort und Übersetzung von Cécile Wajsbrot), Paris, Éditions Zulma, 2008, $496 \mathrm{~S}$.

27 Lorraine Millot, „La RDA in memoriam. Les musées sur l'Allemagne de l'Est se multiplient“, Libération, 15. Januar 1998, S. 28-29. 
unterstrich das Paradoxon: „museale Arbeit soll das Phänomen der Nostalgie verhindern, aber diese gedeiht auch auf dem Felde der Erinnerung" lautete das Fazit.

2010 machte L’Humanité ein Porträt von Maxim Leo, der Titel sprach Klartext: „Das Enkelkind vom Widerstandskämpfer und Antifaschisten Gerhard Leo versucht diesen Menschen besser zu kennen, den er bewundert, aber nicht immer verstanden hat “(28). Anlass war die Herausgabe seines Werkes bei Actes Sud (Histoire d'un Allemand de l'Est), das das Leben der Familie über ein Jahrhundert erzählt. Der Großvater, der die Nazis in Frankreich bekämpfte, war der Pariser Korrespondent vom Organ der SED Neues Deutschland und erfreute sich in der DDR und zu Hause einer großen Ehrfurcht. Er hatte kein Verständnis für die ständigen Klagen über das SED-System, weil er eben einer anderen Generation angehörte. So hielt Maxims Mutter dem Regime die Treue, obwohl die ausgebildete Journalistin die Gleichschaltung nicht billigte, während Leos Vater nie SED-Mitglied gewesen war und sich immer sehr scharf von deren Politik distanzierte. Ein Kommentar des Journalisten fällt dabei in der Einleitung auf: die Mauer, „die die Regierung der DDR früher und auf eigene Initiative hätte abbauen sollen“. Für französische Leser waren solche kritischen Töne im Diskurs der PCF eigentlich neu. Das, was Maxim Leo hier zu retten versuche, sei - so der Journalist - eine politische Kultur, diejenige der Aufbaugeneration, was durchaus der linientreuen Berichterstattung von L'Humanité entsprach, denn Maxim Leo blieb genauso wie seine Mutter Annette Leo, Historikerin, dem SED-System gegenüber sehr kritisch. Eine weitere Behauptung, „die Erinnerung an die mangelnde Meinungsfreiheit, die Schikanen haben weder das Bild der Linken noch die Idee des Sozialismus diskreditiert“, kann dies nur untermauern. Die Kontinuität in der Berichterstattung der französischen Medien zeigt, dass die DDR im deutsch-französischen Kulturraum bei linken Intellektuellen sehr präsent blieb.

\section{Aufarbeitung der DDR-Vergangenheit vs. Ostalgie: die DDR als Alternative?}

Das Interesse der Öffentlichkeit und infolgedessen der Medien für das Phänomen Ostalgie ist nur eine andere Form der Bewertung der ostdeutschen Kultur (im weitesten Sinne des Wortes): sie zielt auch darauf, das Experiment DDR im Sinne einer Alltagsgeschichte und im Gegensatz zur Forschung über das SED-System zu re-legitimieren. W. Thierse forderte 1999, man dürfe „DDR-Bürger nicht gleich als Täter“ einstufen ${ }^{(29)}$. Die französischen Medien riefen bis zur Jahrhundertwende (Goodbye Lenin, 2003) die „Mauer in den Köpfen“ wach, indem sie stets die Worte „Ossis“ und Wessis“ benutzten. Le Figaro hat zwar - wie oben erwähnt - 1999 die Werte der Freiheit und der Marktwirtschaft gepriesen, aber die Zeitung trug auch diesem neuen „Trend“ Rechnung: Ostalgie - diese Begeisterung für DDR-Produkte nach einer Phase des Wegwerfens sei eine „Erfindung des Westens“, sonst würden „Arbeitslose, zurückgesetzte Beamte, ehemalige Stasi-Mitarbeiter“ die PDS wählen, aber nie „von Ostalgie reden“(30).

28 Jean-Paul Pı́́rot, „Le petit fils du résistant antinazi Gerhard Leo cherche à mieux connaître cet homme qu'il admire, mais n'a pas toujours compris“, L’Humanité, 1. Dezember 2010.

29 A. Leparmentier, „Une amnistie“ (Anm. 20).

30 Irina de Chiкоғf, „1989-1999: 1'héritage de l'Est. La RDA très ,tendance““, Le Figaro, 10. November 1999, S. 3 . 
In Libération vertrat die Politologin Sylvie Le Masson 1999 die Ansicht, die DDR sei ja verschwunden, Ostdeutschland aber nicht: es habe sich „als eine unterschiedliche kulturelle und soziale Einheit im Gegensatz zum großen Bruder im Westen“ profiliert; die Ostdeutschen seien keine Nostalgiker, aber infolge der Enttäuschungen, der Desillusionierung „pflegten sie eher einen kritischen Ton gegenüber der Bundesrepublik, die die ex-DDR wirklich unter Vormundschaft gestellt hat “(31). Sie unterstrich somit die Existenz einer Teilöffentlichkeit und in Frankreich muss wahrscheinlich von einer tiefen Resonanz ausgegangen werden. Für sie zeugten die Wahlerfolge der PDS vom „Willen, zu einer neuen Identität zu finden, die auf dem in der DDR Erlebten gründet“. Le Monde bestätigte 2003 den Trend, das „Bedürfnis nach einer nicht erzählbaren Vergangenheit, in dem man sich verorten kann“"(32). 2003 lief nämlich der Film Goodbye Lenin in den Kinos und wurde von „6 Millionen Zuschauern“ gesehen: auch in Frankreich hatte er Erfolg. An der Grenze zwischen Alltagskultur und Nostalgie war für La Croix die Rettung des Ampelmanns erwähnenswert: Die Zeitung skandierte eine Wiedergeburt, da der Ampelmann in Berlin immer noch den Verkehr regeln darf und sogar sehr erfolgreich vermarktet wird ${ }^{(33)}$. AFP stellte auch eine am 23. August 1990 gegründete Ost-Zeitung vor, Super Illu $^{(34)}$, und dokumentierte mit der Darstellung einer geteilten Medienlandschaft die schwierige innere Einigung. Ihre Auflage war nämlich im Osten höher als die der Bild-Zeitung.

Je ferner die Ereignisse rückten, desto glaubwürdiger erschien die These einer anderen Gesellschaftsform. Wie klein aber der Unterschied zwischen Alltagsgeschichte z. B. und Revisionismus ist, zeigte 2009 Krenz' Verteidigungsrede in eigener Sache in L'Humanité: „Die Geschichte wird mich freisprechen“(35).

„Ich gehöre nicht der Generation an, die die Konzentrationslager, den Krieg, den Widerstand, das Exil in Moskau erlebt haben. [...] Ich bin ein Kind der DDR. [...] Mit dem Verschwinden der DDR habe ich einen großen Teil meines Lebens begraben.“

Er stilisierte sich auch in diesem Interview - und der Journalist hat ihm die Gelegenheit dazu gegeben - als Gemäßigter und eben nicht als „Verbrecher“, eine Stigmatisierung, die er aus dem Wiedervereinigungsgebot in der Präambel des Grundgesetzes ableitete und ablehnte. Er rief einen „Leidensweg zahlreicher DDR-Bürger“ wach, die aus ideologischen Gründen „,aus dem Amt entlassen wurden, während es in der DDR keine Arbeitslosigkeit gab “. Auch seien Nazis in der Bundesrepublik so wenig wie möglich geahndet worden, während er ja gewissenhaft die Gesetze der DDR respektierte“, wobei die Argumentation vom Journalisten nicht weiter kommentiert wurde. Als

31 Sylvie Lemasson, „En ex-RDA s'exprime de plus en plus la volonté de se forger une identité pétrie à l’Est. Le temps déverrouillé, Libération, 8. November 1999, S. 5.

32 Georges Marion, „Une vague d',Ostalgie“ frappe l'Allemagne“, Le Monde, 14. August 2003, S. 18. S. auch Patrick SAInt-PAul, „À l’Est, la nostalgie de la République démocratique allemande bat son plein“, Le Figaro, 9. November 2003, S.2 ; Carine Keyvan, „Berlin-Est, un parfum de n-ostalgie...“, Marianne, 17. Oktober 2009, S. 87 (mit einer ausführlichen Liste der ostalgischen Höhepunkte).

33 Blandine Milcent, „Nouvelle jeunesse pour le piéton au chapeau de RDA“, La Croix, 17. Januar 2005, S. 14 .

34 Yannick PASQUET, „Super Illu ou la RDA des starlettes sur papier glacé“, AFP Infos mondiales, 1. Oktober 2010.

35 José FORT, „L'histoire me libérera“ (Interview mit Egon Krenz), L’Humanité, 6. November 2009. 
dann dieser ihn über Gorbatschows Rolle befragte, zögerte er nicht und er bemühte die berühmt-berüchtigte Dolchstoßlegende des deutschen Militärs zur Zeit der Weimarer Republik: „Er hat uns von hinten erdolcht.“ Schließlich gab er zu, dass „das alte System definitiv tot ist. Ich bin gescheitert. Andere müssen jetzt den modernen und demokratischen Sozialismus aufbauen“, denn „Die Zukunft gehört dem Sozialismus, sonst fallen wir in die Barbarei“. Das entsprach durchaus einer in Deutschland und in Frankreich verbreiteten antikapitalistischen Linie (PDS-Die Linke / Lutte ouvrière / le PCF / La France Insoumise).

Im Kontext der Finanz- und Schuldenkrise seit 2008 suchten Menschen nach einer Alternative, was das Experiment DDR in ein neues Licht stellte: so hielt der Trend in den Medien an. 2016 erzählte Le Monde von einer Veranstaltung der Ostalgiker in Bochum, also in Westdeutschland: am 8. Oktober organisierten sie Feierlichkeiten anlässlich des in ihren Augen einzig wichtigen Feiertages, der Gründung der DDR am 7. Oktober 1949. Das Treffen fand in Bochum statt, weil es dort eins der seltenen DDR-Museen in West-Deutschland gibt ${ }^{(36)}$ : tatsächlich darf sonst Berlin als Hochburg des nostalgischen Tourismus betrachtet werden und Berlin ist weltweit „in“ geworden, was das Interesse der französischen Medien auch erklärt. Solche Artikel zeugen dennoch von einem gewissen Verständnis für die sogenannten Staatenlosen. Le Figaro relativierte dies 2009, indem die Tageszeitung sich auf Joachim Gauck bezog, der als Vorsitzender des Vereins Gegen vergessen... für Demokratie vor einem unheilvollen Phänomen warnte: „Sie haben vergessen, dass es keine freien Wahlen gab, keine freien Gewerkschaften, und dass die Partei die Justiz unter Kontrolle hielt“(37). Klaus Schröder konnte es auch 2009 in der Zeitung Le Monde thematisieren ${ }^{(38)}$ : zwar sei Die Linke „eine Volkspartei“ geworden, sie zog eben daraus Nutzen, dass sich Ostdeutsche politisch staatenlos fühlten“, so dass „viele durch die Vereinigung enttäuschte Ostdeutsche und nicht nur überzeugte Sozialisten bzw. Kommunisten für sie stimmten“. Der Aufstieg des linken Populismus, sei es in Frankreich oder in Deutschland, zeigt heute das Bedürfnis nach einer Alternative und so lässt sich auch das eher rege Interesse der französischen Öffentlichkeit für die DDR erklären.

\section{Fazit}

Niemand möchte die DDR retten oder eher wiederhaben: die Delegitimierung der Diktatur wird durchgehend in konservativen Medien behandelt. Das Experiment DDR faszinierte aber noch lange wenigstens eine französische Teilöffentlichkeit: eine gewisse Instrumentalisierung durch Intellektuelle und linke Medien kann feststellt

36 Thomas WIEDER, „Du passé, les nostalgiques de la RDA ne veulent pas faire table rase. Une centaine de personnes se sont retrouvées le 8 octobre à Bochum, dans la Ruhr, pour célébrer la disparue Allemagne de l'Est“, Le Monde, 18. Oktober 2016.

37 P. Saint-Paul, „À l'Est, la nostalgie de la République démocratique allemande bat son plein“ (Anm. 32). Der Artikel betonte in der Einleitung die Aggressivität der Kunden in der Bar „Zur Firma“, nicht weit vom ehemaligen MfS, wenn man sie auf die DDR ansprach.

38 Marie de Verges, „,Peu regrettent le régime, mais il y a une nostalgie à l'égard d'une RDA idéalisée‘. Entretien avec Klaus Schroeder“, Le Monde, 10. November 2009, S.9. K. Schroeder ist Autor einer empirischen Studie über Geschichtskenntnisse der Schüler im Osten und Westen, deren Bilanz verheerend war. 
werden, wie es insbesondere Leitartikel aus der kommunistischen Tageszeitung L'Humanité belegen. Le Monde warb noch 2017 für eine Ausstellung im Berliner Institut Français, „Éclats DDRDA Splitter“(39): ein Historiker, Nicolas Offenstadt, hatte in nicht sanierten Fabriken oder Gebäuden Bilder gemacht und vergessene Gegenstände oder Akten gesammelt (er berief sich dabei auf einen europäischen Trend namens „Urbex“). Die Verwüstung der ehemaligen Fabriken oder Behörden zeuge seines Erachtens von einem Ausverkauf der DDR, einer Delegitimierung, und Libération integrierte kurz darauf diese Analyse in die Debatte um die rechtsradikalen Verfolgungen in Chemnitz ${ }^{(40)}$. Dabei sind brachliegende Gebäude eigentlich typisch für umstrukturierte Industriereviere, egal ob in Ost-Berlin, Essen oder Roubaix (F). Der Artikel sorgte für Polemik: Libération gab dann einem anderen Wissenschaftler das Wort, der die Thesen von Offenstadt widerlegte ${ }^{(41)}$. Im Bereich der Aufarbeitung der DDR-Geschichte herrscht auch in Frankreich nicht immer Einstimmigkeit unter französischen Forschern.

Generell stimmten die französischen Medien also doch darin überein, dass nicht alles falsch gewesen sei, was in der DDR existierte. Im Kontext des dreißigsten Jahrestages des Mauerfalls soll die Oral History, die Geschichte der Zeugen, nicht nur der Opfer, darüber Bericht erstatten. Vom Fazit ausgehend, Public History würde im Bereich der DDR-Forschung „boomen“, wollen junge deutsche Forscher neue Wege gehen, wie es die Thematik folgenden Forschungsprojektes zeigt: „Es ist nicht alles gesagt. Ein Workshop zur DDR-Forschung - Die Frage nach der Zukunft der DDRForschung hat Konjunktur, gerade mit Blick auf das Gedenkjahr 2019 - Wir feiern 30 Jahre Friedliche Revolution “(42). Französische Medien waren oft bemüht und werden noch 2019 wahrscheinlich bemüht sein, ein nuanciertes Bild der inneren Einheit zu geben, d. h. auch der deutschen Gesellschaft seit der Wiedervereinigung: die Regelmäßigkeit, mit der über die DDR berichtet wird, zeugt von einem kaum nachlassenden Interesse für einen gesellschaftlichen Idealtypus. Im Kontext einer europäischen Geschichtspolitik und der Post Cold War Studies blieb die DDR wenigstens 1990 bis 2009 im deutsch-französischen bzw. europäischen öffentlichen Raum sehr präsent. Neue Ereignisse und Entwicklungen sorgen für Aufklärungsbedürfnis: im Kontext des Aufstiegs der AfD und insbesondere der Ereignisse in Chemnitz im Sommer 2018 veröffentlichte sogar die Sportzeitung L'Équipe in ihrem Wochenendmagazin ein Dossier über Verbindungen zwischen Fußballvereinen der DDR und Neonazis, namentlich ihren Fans und Legida ${ }^{(43)}$.

39 Thomas Wieder, „Sur les traces de la RDA“, Le Monde, 3. Juni 2017, S. 6.

40 Catherine CALvet, „Nicolas Offenstadt: Les lieux abandonnés de la RDA montrent à quel point elle a été délégitimée (Interview)“, Libération, 11. September 2018.

41 Jean-Louis GEORGET, „L’historien et le terrain: retour en RDA“, Libération, 18. September 2018.

42 Steffi BrünIng, Universität Rostock; Maria Neumann, Humboldt Universität, 30.11.2018-01.12.2018, Berlin, Institut für Geschichtswissenschaften, Humboldt-Universität.

43 Françoise InIzan, „Sombres nuages sur le ,bleu ciel“ de Chemnitz“, L’Équipe, 22. September 2018, S. 40-46. Blau ist auch die Farbe des Chemnitzer Fussballclubs (CFC). Die rechtsradikale Hooligansgruppe nennt sich Kaotik Chemnitz. LEGIDA (Leipzig) ist das Pendant zu PEGIDA (Dresden). 


\section{Résumé}

Depuis le traité de l'Élysée signé en 1963 par de Gaulle et Adenauer, les relations franco-allemandes étaient avant tout les relations entre la France et la République fédérale. À l'instar de François Mauriac, ministre de De Gaulle, qui disait "J'aime tellement l'Allemagne que je préfère qu'il y en ait deux", une partie de l'opinion publique a considéré la deuxième Allemagne comme une alternative ou un modèle jusqu'en 1989. Dans le contexte de la Guerre froide, il existait un contre-espace public autour du PCF et d'une partie du PS. En 1989, certains Français n'étaient pas prêts à voir disparaître la RDA, une expérience placée sous le signe de l'antifascisme et du communisme, et ils ne doutaient pas de sa capacité à se réformer. De 1990 à 2009, sauver la RDA signifierait donc ouvrer pour un devoir de mémoire, qui ne serait pas seulement axé autour de la mémoire de la dictature et de la délégitimation du système-SED.

Cette étude s'articule autour des problématiques de la victoire du monde libre et de la société capitaliste, des questions culturelles et de mémoire avec une mise en perspective des opinions publiques française et allemandes et une analyse des sous-espaces publics ainsi structurés: pour le corpus d'articles de presse ont été privilégiées les années 2000 avec la commémoration des dix ans de l'unité et un premier bilan de l'unification, et 2009 avec la commémoration des soixante ans de la création de la RFA et des vingt ans de la chute du mur, ainsi qu'un débat sur la RDA comme État de non-droit. L'étude met en évidence que la RDA a vraiment occupé une place spécifique dans l'espace public européen et permet de vérifier à travers l'information la persistance d'une nostalgie du communisme en France.

\footnotetext{
Abstract

Since the signing of the Elysée Treaty in 1963, the Franco-German relationship was above all about France and the Federal Republic. One of de Gaulle's Minister, François Mauriac, said: "I love Germany so much I'm glad there are two of them" and so thought a part of the public opinion who considered the GDR an alternative or a model until 1989. Back then, some French were not ready for the demise of the GDR, its antifascism and communism. From 1990 to 2009, saving the GDR also meant working for a duty of remembrance, which would not only focus on the memory of the dictatorship and the delegitimation of the SED system.

Putting in perspective the French and German public opinions on a public sub-space level, this study addresses the following two issues: the victory of the Free World and the capitalist society and the cultural questions about memory. The corpus favours the 2000 s - and especially the first evaluation of the unification upon the commemoration of the ten years of unity - and 2009, covering the commemorations of the FRG creation and the fall of the Berlin wall as well as a debate on the GDR as a state of lawlessness. It highlights the specific place of the GDR in the European public space and corroborates that France looked back on communism with nostalgia.
} 